\title{
Autoparallels From a New Action Principle
}

\author{
H. Kleinert and A. Pelster \\ Institut für Theoretische Physik, Freie Universität Berlin, Arnimallee 14, 14195 Berlin, Germany
}

(October 7, 2018)

We present a simpler and more powerful version of the recently-discovered action principle for the motion of a spinless point particle in spacetimes with curvature and torsion. The surprising feature of the new principle is that an action involving only the metric can produce an equation of motion with a torsion force, thus changing geodesics to autoparallels. This additional torsion force arises from a noncommutativity of variations with parameter derivatives of the paths due to the closure failure of parallelograms in the presence of torsion.

According to Einstein's equivalence principle, the equation of motion of a free spinless point particle in a curved spacetime is found by the following two step procedure. First, the equation of motion

$$
\ddot{x}^{a}(\tau)=0
$$

for rectilinear coordinates $x^{a}(a=0,1,2,3)$ in a flat spacetime with the Minkowski metric $\left(\eta_{a b}\right)=(+,-,-,-)$ is transformed via

$$
x^{a}=x^{a}(q)
$$

to curvilinear coordinates $q^{\lambda}(\lambda=0,1,2,3)$. The resulting equation reads

$$
\ddot{q}^{\lambda}(\tau)+\bar{\Gamma}_{\mu \nu}^{\lambda}(q(\tau)) \dot{q}^{\mu}(\tau) \dot{q}^{\nu}(\tau)=0,
$$

where $\tau$ is the proper time, and the Christoffel connection

$$
\begin{aligned}
& \bar{\Gamma}_{\mu \nu}^{\lambda}(q)=\frac{1}{2} g^{\lambda \kappa}(q) \\
& \times\left[\partial_{\mu} g_{\nu \kappa}(q)+\partial_{\nu} g_{\kappa \mu}(q)-\partial_{\kappa} g_{\mu \nu}(q)\right]
\end{aligned}
$$

is derived from the induced metric

$$
\begin{aligned}
g_{\mu \nu}(q) & =e^{a}{ }_{\mu}(q) e^{b}{ }_{\nu}(q) \eta_{a b}, \\
e^{a}{ }_{\lambda}(q) & \equiv \partial x^{a}(q) / \partial q^{\lambda} .
\end{aligned}
$$

Second, it is postulated that (3) and (14) also describe the motion in a curved spacetime with an intrinsic metric $g_{\mu \nu}$. The solutions of (3) represent the shortest curves in the spacetime, i.e. geodesics.

If torsion is admitted to the geometry, thus generalizing the Riemann to a Cartan spacetime [1], there exists another equation of motion, which is as covariant and simple as (3),

$$
\ddot{q}^{\lambda}(\tau)+\Gamma_{\mu \nu}^{\lambda}(q(\tau)) \dot{q}^{\mu}(\tau) \dot{q}^{\nu}(\tau)=0,
$$

in which the Christoffel connection $\bar{\Gamma}_{\mu \nu}{ }^{\lambda}$ is replaced by the full affine or Cartan connection

$$
\Gamma_{\mu \nu}^{\lambda}(q)=\bar{\Gamma}_{\mu \nu}^{\lambda}(q)+K_{\mu \nu}^{\lambda}(q) .
$$

It contains in addition to the Christoffel connection (4) the contortion tensor

$$
K_{\mu \nu}^{\lambda}(q)=S_{\mu \nu}^{\lambda}(q)-S_{\nu \mu}^{\lambda}(q)+S_{\mu \nu}^{\lambda}(q),
$$

a combination of torsion tensors

$$
S_{\mu \nu}^{\lambda}(q) \equiv \frac{1}{2}\left[\Gamma_{\mu \nu}^{\lambda}(q)-\Gamma_{\nu \mu}^{\lambda}(q)\right] .
$$

The solutions of (7) define the straightest curves in the spacetime, i.e. autoparallels. Note that due to (8) and (9) the autoparallels (7) and the geodesics (3) differ by the additional torsion force $K_{\mu \nu}{ }^{\lambda}(q) \dot{q}^{\mu} \dot{q}^{\nu}=2 S^{\lambda}{ }_{\mu \nu}(q) \dot{q}^{\mu} \dot{q}^{\nu}$. It should be noted, that the symmetric part $K_{(\mu \nu)}{ }^{\lambda}$ of the contorsion tensor does not identically vanish.

The question arises which of the two curves provides us with the correct particle trajectories in spacetimes with curvature and torsion. According to the Einstein-Cartan theory of gravity [2], the canonical energy-momentum tensor $\Theta^{\mu \nu}$ and the spin density $\Sigma^{\mu \nu, \lambda}$ of matter should determine the geometry of spacetime by equations $G^{\mu \nu}=-\kappa \Theta^{\mu \nu}$ and $S^{\mu \nu, \lambda}=-\kappa \Sigma^{\mu \nu, \lambda}$, where $\kappa$ is the gravitational constant, $S^{\mu \nu, \lambda}$ the Palatini tensor derived from the torsion tensor $S_{\mu \nu}{ }^{\lambda}$ and $G^{\mu \nu}$ the Einstein tensor formed from the Cartan curvature tensor

$$
\begin{aligned}
R_{\mu \nu \kappa}{ }^{\lambda} & (q)=\partial_{\mu} \Gamma_{\nu \kappa}^{\lambda}(q)-\partial_{\nu} \Gamma_{\mu \kappa}{ }^{\lambda}(q) \\
& -\Gamma_{\mu \kappa}{ }^{\rho}(q) \Gamma_{\nu \rho}{ }^{\lambda}(q)+\Gamma_{\nu \kappa}{ }^{\rho}(q) \Gamma_{\mu \rho}{ }^{\lambda}(q) .
\end{aligned}
$$


The Bianchi identity for the Einstein tensor $G^{\mu \nu}$ implies for the symmetric energymomentum tensor $T^{\mu \nu}$ of spinless point particles the conservation law

$$
\bar{D}_{\nu} T^{\mu \nu}(q)=0,
$$

where $\bar{D}_{\nu}$ is the covariant derivative involving the Christoffel connection $\bar{\Gamma}_{\mu \nu}^{\lambda}$. This conservation law does not contain the torsion tensor $S_{\mu \nu}{ }^{\lambda}$, and leads therefore directly to the geodesic equation (3) as shown by Hehl [5].

Because of the beauty of the mathematical framework within which this result was derived, there was little doubt that particles should run along the shortest paths, just as in Einstein's original theory of gravity. Physically, however, it is hard to conceive how this can be true, since it contradicts two quite fundamental properties of physical laws: inertia and locality. Because of its inertia, a particle will change its direction in a minimal way at each instant of time, which makes its trajectory as straight as possible. If it were to choose a path which minimizes the length of the orbit it would have possessed some global information of the geometry. In Einstein's theory of gravity, the two paths happen to coincide on mathematical grounds, so that this basic problem did not become apparent for geodesics, but in the presence of torsion it can no longer be ignored.

Doubts as to the correctness of geodesics as particle trajectories arose first in a completely different context [6,7]. When solving nonrelativistic Coulomb problems in classical or quantum mechanics, a local coordinate transformation

$$
d x^{a}=e_{\lambda}^{a}(q) d q^{\lambda}
$$

with the Kustaanheimo-Stiefel coefficients 8.99

$$
\left(e_{\lambda}^{a}(q)\right)=\left(\begin{array}{rrrr}
q^{3} & q^{0} & q^{1} & q^{2} \\
q^{0} & -q^{3} & -q^{2} & q^{1} \\
q^{1} & q^{2} & -q^{3} & -q^{0} \\
q^{2} & -q^{1} & q^{0} & -q^{3}
\end{array}\right)
$$

has long been very helpful since it carries Coulomb into harmonic systems. The coefficient functions $e_{\lambda}^{a}$ possess the interesting property of not obeying the integrability condition of Schwarz:

$$
\partial_{\mu} e_{\lambda}^{a}(q)-\partial_{\lambda} e_{\mu}^{a}(q) \neq 0
$$

This implies that there exists no singlevalued global transformation (2) from which $e_{\lambda}^{a}$ could be obtained as the derivatives (6). This makes the local coordinate transformation (13) nonholonomic. In the absence of forces in the original flat space, particles run along straight lines which satisfy the equation (11). Their image under the transformation (13) satisfies the autoparallel equation (7), where the affine connection is explicitly given by

$$
\Gamma_{\mu \nu}^{\lambda}(q)=e_{a}^{\lambda}(q) \partial_{\mu} e^{a}{ }_{\nu}(q) .
$$

From (10), (11) and (16) we then conclude that a coordinate transformation of the type (13) carries a flat space into a space with the torsion tensor

$$
S_{\mu \nu}^{\lambda}(q)=\frac{1}{2} e^{a}{ }_{\lambda}(q)\left[\partial_{\mu} e^{a}{ }_{\nu}(q)-\partial_{\nu} e^{a}{ }_{\mu}(q)\right]
$$

and a vanishing Cartan curvature tensor [7]. Now, it is well-known that equations of motion remain valid under such mappings. Therefore autoparallels must be the correct particle trajectories [6],7].

It is not hard to generate also nonvanishing Cartan curvature by nonholonomic mappings (13). For this, the coefficient functions $e^{a}{ }_{\lambda}$ must only be chosen as multivalued. Then the functions $e^{a}{ }_{\lambda}$ themselves fail to satisfy the criterion of Schwarz, and the noncommutativity of partial derivatives yields for the Cartan curvature tensor (11) the expression

$$
R_{\mu \nu \lambda}^{\kappa}(q)=e_{a}^{\kappa}(q)\left[\partial_{\mu} \partial_{\nu}-\partial_{\nu} \partial_{\mu}\right] e_{\lambda}^{a}(q),
$$

as can be verified by inserting (16). Local coordinate transformations (13) which generate both torsion (17) and curvature (18) are widely used for describing crystals with defects 10 13. Examples illustrating such mappings and their applications are elaborated in [3].

In Ref. [14] it was pointed out that autoparallel trajectories could only be understood after a revision of the variational calculus in spacetimes with torsion. These possess an unusual feature not encountered before, namely a closure failure of parallelograms. As a consequence, variations of particle trajectories in an action cannot be performed as usual. Variations $\delta x^{a}(\tau)$ of a path in flat spacetime are always performed at 
vanishing endpoints, thus forming closed paths. The images $\delta q^{\lambda}(\tau)$ under a nonholonomic mapping (13), however, are in general open, their closure failure being proportional to the torsion. The superscript $S$ indicates this special feature.

Let us briefly recall the derivation in [14. If $e_{a}{ }^{\lambda}$ denotes the reciprocal multivalued basis vectors orthonormal to $e_{\mu}^{a}$,

$$
e_{a}^{\lambda}(q) e_{\mu}^{a}(q)=\delta_{\mu}^{\lambda},
$$

the local coordinate transformation $(13)$ can be integrated implicitly for any orbit $x^{a}(\tau)$ in the flat spacetime to yield an implicit equation for the image orbit $q^{\lambda}(\tau)$ in the spacetime with curvature and torsion:

$q^{\lambda}(\tau)=q^{\lambda}\left(\tau_{0}\right)+\int_{\tau_{0}}^{\tau} d \tau^{\prime} e_{a}^{\lambda}\left(q\left(\tau^{\prime}\right)\right) \dot{x}^{a}\left(\tau^{\prime}\right)$

If $\delta x^{a}(\tau)$ denotes an arbitrary variation of the orbits in the flat spacetime, nonholonomic variations $\delta^{S} q^{\lambda}(\tau)$ are defined by identifying the image of the varied path $x^{a}(\tau)+\delta x^{a}(\tau)$ under the mapping (20) with $q^{\lambda}(\tau)+\delta^{S} q^{\lambda}(\tau)$. The variations $\delta x^{a}(\tau)$ are performed as usual at fixed end points. Their images $\delta^{S} q^{\lambda}(\tau)$, however, possess the above-mentioned closure failure which is proportional to the amount of torsion introduced inside $\delta^{S} q^{\lambda}(\tau)$ by the nonholonomic coordinate transformation (13). They are open nonholonomic variations which may be chosen to vanish at the initial point, but then they are nonzero at the final point. Note that the open nonholonomic variations $\delta^{S}$ commute with the parameter derivatives $d_{\tau} \equiv \partial / \partial \tau$ :

$$
\delta^{S} d_{\tau} q^{\lambda}-d_{\tau} \delta^{S} q^{\lambda}=0
$$

Applying these variations to an action

$$
\mathcal{A}\left[q^{\lambda}(\tau)\right]=\int_{\tau_{1}}^{\tau_{2}} d \tau L(q(\tau), \dot{q}(\tau)),
$$

the correct variational principle in the presence of curvature and torsion was found in [14 to have the form

$$
\delta^{S} \mathcal{A}\left[q^{\lambda}(\tau)\right]=0 .
$$

Applying this new variational principle to the Lagrangian of a spinless point particle

$$
L(q, \dot{q})=-M c \sqrt{g_{\lambda \mu}(q) \dot{q}^{\lambda} \dot{q}^{\mu}}
$$

produces directly the autoparallel equation (7).

In spite of the simplicity of the result, the algebra involved in deriving the torsion terms in the equation of motion (7) turned out to be quite complicated 14. In addition, the applicability of the procedure was restricted to a free particle Lagrangian, and did not permit the inclusion of nongeometric forces such as electromagnetic ones. The purpose of this note is to improve this situation by presenting a variational procedure which is more elegant and can be applied to general Lagrangians. This is possible by avoiding the awkward open anholonomic variations $\delta^{S} q^{\mu}(\tau)$ in favor of auxiliary closed nonholonomic variations $\delta$ which do vanish at the endpoints, in this respect being closer to the ordinary variations. They are defined as the images of the ordinary variations $\delta x^{a}(\tau)$ in the flat spacetime under the local coordinate transformation (13):

$$
\delta q^{\mu}(\tau) \equiv e_{a}^{\mu}(q(\tau)) \delta x^{a}(\tau) .
$$

Their special property, which will generate the torsion force later on, is that they do not commute with parameter derivatives of the path functions $q^{\mu}(\tau)$. To see this we invert (25) and take the parameter derivative to obtain

$$
d_{\tau} \delta x^{a}=e_{\lambda}^{a}(q) d_{\tau} \delta q^{\lambda}+\partial_{\mu} e_{\lambda}^{a}(q) \dot{q}^{\mu} \delta q^{\lambda} .
$$

A variation of (13) yields, on the other hand,

$$
\delta d_{\tau} x^{a}=e_{\lambda}^{a}(q) \delta \dot{q}^{\lambda}+\partial_{\mu} e_{\lambda}^{a}(q) \dot{q}^{\lambda} \delta q^{\mu} .
$$

Using now the property

$$
\delta d_{\tau} x^{a}-d_{\tau} \delta x^{a}=0
$$

in flat spacetime, we deduce from (26) and (27) that the operations $\delta$ and $d_{\tau}$ satisfy the commutation relation

$$
\delta d_{\tau} q^{\lambda}-d_{\tau} \delta q^{\lambda}=2 S_{\mu \nu}^{\lambda}(q) \dot{q}^{\mu} \delta q^{\nu} .
$$

The new action principle states now

$$
\delta \mathcal{A}\left[q^{\lambda}(\tau)\right]=0
$$

for all variations $\delta q^{\lambda}(\tau)$ which vanish at the end points $\tau_{1}$ and $\tau_{2}$,

$$
\delta q^{\lambda}\left(\tau_{1}\right)=\delta q^{\lambda}\left(\tau_{2}\right)=0,
$$


and which satisfy the commutation relation (29). The latter property of the auxiliary variations in spacetimes with torsion modifies the Euler-Lagrange equations by the correct torsion force. Indeed, varying the action (22) by $\delta q^{\lambda}(\tau)$ yields

$$
\begin{aligned}
& \delta \mathcal{A}\left[q^{\lambda}(\tau)\right]= \\
& =\int_{\tau_{1}}^{\tau_{2}} d \tau\left[\frac{\partial L}{\partial q^{\lambda}(\tau)} \delta q^{\lambda}(\tau)+\frac{\partial L}{\partial \dot{q}^{\lambda}(\tau)} \frac{d}{d \tau} \delta q^{\lambda}(\tau)\right. \\
& \left.\quad+2 S_{\mu \nu}{ }^{\lambda}(q(\tau)) \frac{\partial L}{\partial \dot{q}^{\lambda}(\tau)} \dot{q}^{\mu}(\tau) \delta q^{\nu}(\tau)\right]
\end{aligned}
$$

After a partial integration of the second term, we obtain the modified Euler-Lagrange equation

$$
\begin{array}{r}
\frac{\partial L}{\partial q^{\lambda}(\tau)}-\frac{d}{d t} \frac{\partial L}{\partial \dot{q}^{\lambda}(\tau)} \\
=2 S_{\lambda \mu}{ }^{\nu}(q(\tau)) \dot{q}^{\mu}(\tau) \frac{\partial L}{\partial \dot{q}^{\nu}(\tau)},
\end{array}
$$

the right-hand side being the torsion force.

For the free-particle Lagrangian (24), the equation of motion (33) takes the form

$$
\begin{array}{r}
\ddot{q}^{\lambda}\left[g ^ { \lambda \kappa } \frac { 1 } { 2 } \left(\partial_{\mu} g_{\nu \kappa}+\partial_{\nu} g_{\kappa \mu}\right.\right. \\
\left.\left.-\partial_{\kappa} g_{\mu \nu}\right)+2 S_{\mu \nu}^{\lambda}\right] \dot{q}^{\mu} \dot{q}^{\nu}=0
\end{array}
$$

which coincides with the autoparallel equation (7) after taking into account (4), (8) and (9). Thus spinless point particles move along straightest lines, as expected from particle inertia and locality, rather than shortest lines, as commonly believed. Furthermore we observe the increased power of the new variational principle (30). The modified Euler-Lagrange equation (33) make also sense if the Lagrangian contains a coupling to nongeometric fields, as for instance the vector potential $A_{\mu}$.

The new action principle presented here is the most natural generalization of the fundamental Hamilton principle to spacetimes with curvature and torsion. In contrast to [14], variations can be performed without reference to the flat coordinate system, which was only introduced as a mathematical device for deriving the crucial commutation relation (29) between variation and parameter derivative.
Since spinless point particles run along autoparallel trajectories on very fundamental grounds , i.e. inertia, locality and closure failure, the gravitational field equations in spaces with torsion, on which the presently accepted derivation of geodesic trajectories is based, must have a fundamental flaw. In particlular, they must be such that the covariant conservation law for the energy-momentum tensor of the point particle is

$$
\bar{D}_{\nu} T^{\mu \nu}+2 S_{\lambda \kappa}^{\mu} T^{\kappa \lambda}=0,
$$

instead of (12). This will be an important task for the future.

[1] J.A. Schouten: Ricci-Calculus; Springer, Second Edition (1954).

[2] F.W. Hehl, P. von der Heyde, G.D. Kerlick, J.M. Nester, Rev. Mod. Phys. 48, 393, 1976.

[3] H. Kleinert: Gauge Fields in Condensed Matter, Vol. II, Part IV, Differential Geometry of Defects and Gravity; World Scientific (1989).

[4] F.W. Hehl, J.D. McCrea, E.W. Mielke, Y. Ne'eman, Phys. Rep. 258, 1, 1995.

[5] F.W. Hehl, Phys. Lett. A 36, 225, 1971.

[6] H. Kleinert, Mod. Phys. Lett. A 4, 2329, 1989; first comprehensively discussed in the 1990 edition of the textbook 汭.

[7] H. Kleinert, Path Integrals in Quantum Mechanics, Statistic and Polymer Physics; World Scientific, Second Edition (1995).

[8] P. Kustaanheimo, E. Stiefel, J. Reine Angew. Math. 218, 204, 1965.

[9] E. L. Stiefel and G. Scheifele, Linear and Regular Celestial Mechanics, Springer, Berlin (1971).

[10] K. Kondo, Lecture in: Proc. 2nd Japan Nat. Congr. Applied Mechanics, Tokio (1952).

[11] B.A. Bilby, R. Bullough, E. Smith, Proc. R. Soc. London A 231, 263, 1955.

[12] E. Kröner: Continuum Theory of Defects; In: R. Balian et al., Physics of Defects, Les Houches, Session XXXV, 1980, NorthHolland (1981).

[13] E. Kröner, Intern. J. Theor. Phys. 29, 1219, 1990.

[14] P. Fiziev, H. Kleinert, Europhys. Lett. 35, 241, 1996 (hep-th/9503074). 
[15] P. Fiziev, H. Kleinert, Euler Equations for Rigid Body - A Case for Autoparallel Trajectories in Spaces with Torsion, hep-th/9503075.

\section{Note added in proof:}

Between the writing of this letter and its final print in Gen. Rel. Grav. 31, 1439 (1999), numerous new results have been found. For gradient torsion, gravitational field equations have been set up $[1,2,3]$ whose semiclassical trajectories are autoparallels [4]. However, it seems to be impossible to construct a realistic field theory with a general torsion field. First, there are serious consistency problems of the coupling of massless versus massive vector mesons to torsion [4]. Second, field theories with general torsion seem to violate a basic universality principle of angular and spin momentum in elementary particle physics [5].

[1] H. Kleinert, Nonholonomic Mapping Principle for Classical and Quantum Mechanics in Spaces with Curvature and Torsion (aps1997sep03 002).

[2] H. Kleinert, Act. Phys. Pol. B 29, 1033 (1998) (gr-qc/9801003).

[3] H. Kleinert, A. Pelster, Act. Phys. Pol. B 29, 1015 (1998) (gr-qc/9801030).

[4] H. Kleinert, Phys. Let B 440, 283 (1998) (gr-qc/9808022).

[5] H. Kleinert, Universality Principle for Orbital Angular (gr-qc/9807021). 